\title{
BMJ Open Relationship between serum uric acid and clustering of cardiovascular disease risk factors and renal disorders among Shanghai population: a multicentre and cross-sectional study
}

Min Tao, ${ }^{1}$ Xiaoling Pi, ${ }^{2}$ Xiaoyan Ma, ${ }^{1,3}$ Yingfeng Shi, ${ }^{1}$ Yuzhen Zhang, ${ }^{4}$ Hongwei Gu, ${ }^{1}$ Yongbin Chi, ${ }^{5}$ Shougang Zhuang, ${ }^{1,6} \mathrm{Na} \mathrm{Liu}^{7}$

To cite: Tao M, Pi X, Ma X, et al. Relationship between serum uric acid and clustering of cardiovascular disease risk factors and renal disorders among Shanghai population: a multicentre and crosssectional study. BMJ Open 2019;9:e025453. doi:10.1136/ bmjopen-2018-025453

\section{- Prepublication history for} this paper is available online. To view these files, please visit the journal online (http://dx.doi org/10.1136/bmjopen-2018025453).

MT, XP and XM contributed equally.

Received 25 August 2018 Revised 29 December 2018 Accepted 21 January 2019

Check for updates

(C) Author(s) (or their employer(s)) 2019. Re-use permitted under CC BY-NC. No commercial re-use. See rights and permissions. Published by BMJ.

For numbered affiliations see end of article.

Correspondence to

Professor Na Liu;

naliubrown@163.com

\section{ABSTRACT}

Objectives To estimate the current prevalence of cardiovascular disease risk factors (CRFs) and renal disorders across serum uric acid (SUA) quartiles, and evaluate the relationships between SUA and CRFs and renal diseases in Shanghai population.

Study design Observational, cross-sectional study. Setting Data were obtained from the physical check-up of local residents at three hospitals in Shanghai.

Participants Residents were invited to take part in a physical check-up and provided informed consent. Exclusion criteria were diseases that resemble cancer, hepatic disease, and other coexisting illnesses including autoimmune kidney diseases and renal artery stenosis, individuals treated with xanthine oxidase inhibitors, and those with incomplete information. There are 26768 individuals in our study.

\section{Primary and secondary outcome}

measures Hyperuricaemia was defined as SUA $\geq 7 \mathrm{mg} / \mathrm{dL}$ in men and $\geq 6 \mathrm{mg} / \mathrm{dL}$ in women or taking xanthine oxidase inhibitors. Subjects were divided into gender-specific quartiles. We estimate the prevalence of CRFs and renal disorders across SUA quartiles. The relationships between SUA and CRFs and renal disorders in both genders were evaluated using logistic regression analysis.

Results There was a significant increase in the prevalence of major CRFs and renal diseases across SUA quartiles in a separate analysis among men and women (all $\mathrm{p}$ trend $<0.001$ ). After multiple adjustment, hyperuricaemia positively correlated with obesity (male $\mathrm{OR}=3.165, \mathrm{p}<0.001$; female $\mathrm{OR}=3.776, \mathrm{p}<0.001$ ), hypertension (male $\mathrm{OR}=1.341, \mathrm{p}<0.001$; female $\mathrm{OR}=1.289, \mathrm{p}=0.006$ ), dyslipidaemia (male $\mathrm{OR}=2.490$, $\mathrm{p}<0.001$; female $\mathrm{OR}=3.614, \mathrm{p}<0.001$ ), chronic kidney disease (male $0 R=7.081, p<0.001$; female $O R=11.571$, $\mathrm{p}<0.001$ ) and nephrolithiasis (male $\mathrm{OR}=1.469, \mathrm{p}<0.001$; female $O R=1.242, p=0.041$ ), but negatively correlated with diabetes mellitus (male $\mathrm{OR}=0.206, p<0.001$; female $\mathrm{OR}=0.524, p<0.001$ ). There was a stronger association between hyperuricaemia and clustered CRFs as well as chronic kidney disease in women than in men.

Conclusions In Shanghai population, concomitant with the elevated level of SUA, the prevalence of CRFs and renal diseases was rising. Hyperuricaemia was significantly
Strengths and limitations of this study

- The study used strict exclusion criteria based on medical histories and laboratory findings.

- We conducted a multicentre study with a large sample size, which ensured sufficient power in obtaining an accurate rate of prevalence of cardiovascular disease risk factors (CRFs) and renal diseases, and in analysing the relationship between serum uric acid and CRFs and renal disorders across serum uric acid quartiles.

- The relationship was analysed in both genders, and we got a solid conclusion on the differences between men and women.

- It was a cross-sectional study and the results could not establish causative relationships between hyperuricaemia and CRF clustering and renal diseases.

- Data were from three medical centres' databases which lacked details on waist circumference, homeostasis model assessment of insulin resistance, smoking, drinking, lifestyles, diet and pharmacotherapy, which might affect deviations in some clinical outcomes.

associated with CRFs and renal disorders, especially in women.

\section{INTRODUCTION}

Uric acid (UA) is the final degradation product of purine metabolism in the liver, muscles and intestines. ${ }^{1}$ A high level of serum uric acid (SUA) is correlated with multiple disorders such as metabolic syndrome, cardiovascular disease (CVD) as well as kidney diseases..$^{2-4}$ The association between hyperuricaemia and cardiovascular disease risk factors (CRFs) has been widely focused since the last century. ${ }^{5}$ There are various risk factors involved in CVDs, including age, sex, obesity, hypertension, diabetes mellitus, 
dyslipidaemia, family history, smoking, depression and so on.$^{6-8}$ Numerous indexes of CRFs were closely associated with increased SUA, such as body mass index (BMI), cholesterol, triglyceride (TG), low-density lipoprotein cholesterol (LDL-C), high-density lipoprotein cholesterol (HDL-C), systolic blood pressure (SBP), diastolic blood pressure (DBP) and fasting plasma glucose (FPG). ${ }^{6} 79$ However, the relationship between hyperuricaemia and CRFs in both genders of the Shanghai population has not been well studied, and data from multiple clinical centres in China are extremely limited. In this study, we evaluated the prevalence of major CRFs (obesity, hypertension, diabetes mellitus and dyslipidaemia) across SUA quartiles and analysed the association of these factors with SUA level respectively in both men and women.

It has been documented that $70 \%$ of the daily UA production is excreted by the kidney. ${ }^{10}$ UA tends to crystallise in low urine $\mathrm{pH}$. Hyperuricaemia reduces urine $\mathrm{pH}$ and increases the risk of formation of urate stones. ${ }^{11}$ Recent research indicated that SUA level could predict the development of albuminuria, ${ }^{12}{ }^{13}$ and elevated SUA level was significantly associated with estimated glomerular filtration rate (eGFR) decline. ${ }^{1415}$ However, whether UA is a cause or associated with renal diseases is a question that still awaits further investigations. Thus, we assessed the prevalence of renal diseases across SUA quartiles, and the relationship between SUA and renal disorders in Shanghai population.

\section{METHODS}

\section{Study population}

Permanent residents aged between 16 and 98 years who participated in health check-up during the period from January 2015 and December 2015 in three medical centres (Shanghai East Hospital Affiliated to Tongji University School of Medicine, Pudong New District Gongli Hospital and Baoshan Branch of Shanghai First People's Hospital) were invited to the study. After excluding subjects with incomplete data, and those with cancer, hepatic disease or other coexisting illnesses including autoimmune kidney diseases and renal artery stenosis, as well as subjects treated with xanthine oxidase inhibitors, 26768 participants were enrolled in our study.

\section{Primary outcome}

Hyperuricaemia was defined as SUA $\geq 7 \mathrm{mg} / \mathrm{dL}$ in men and $\geq 6 \mathrm{mg} / \mathrm{dL}$ in women or current use of xanthine oxidase inhibitors. ${ }^{16}$ SUA was determined using the uricase-peroxidase method.

\section{Study definitions}

BMI was calculated as weight in kilograms divided by height in squared metres. According to the WHO guidelines for the Asia-Pacific population, normal weight was defined as $18.5 \leq \mathrm{BMI}<24.0 \mathrm{~kg} / \mathrm{m}^{2}$, overweight was defined as $24.0 \leq \mathrm{BMI}<28.0 \mathrm{~kg} / \mathrm{m}^{2}$, obesity was defined as BMI $\geq 28.0 \mathrm{~kg} / \mathrm{m}^{2}$, and underweight was defined as
BMI $<18.5 \mathrm{~kg} / \mathrm{m}^{2} .{ }^{17}$ Blood pressure (BP) measurements were taken according to the Joint National Committee VII criteria. ${ }^{18}$ Normal BP was defined as having SBP $<120 \mathrm{~mm}$ $\mathrm{Hg}$ and DBP $<80 \mathrm{~mm} \mathrm{Hg}$. Prehypertension was defined as having an SBP of $120-139 \mathrm{~mm} \mathrm{Hg}$ and/or DBP of $80-89 \mathrm{~mm} \mathrm{Hg}$. Grade 1 hypertension was defined as having an SBP of 140-159 mm Hg and/or DBP of 90-99 mm Hg. Grade 2 or grade 3 hypertension was defined as an SBP $\geq 160 \mathrm{~mm} \mathrm{Hg}$ and/or DBP $\geq 100 \mathrm{~mm} \mathrm{Hg} .{ }^{18}$ According to the Chinese adult dyslipidaemia prevention guide (2007 edition), fasting total cholesterol (TC) $\geq 6.22 \mathrm{mmol} / \mathrm{L}$, TG $\geq 2.26 \mathrm{mmol} / \mathrm{L}, \quad$ HDL-C $<1.04 \mathrm{mmol} / \mathrm{L}$ and $/$ or LDL-C $>4.14 \mathrm{mmol} / \mathrm{L}$, or currently undergoing pharmacological treatment, were defined as dyslipidaemia. ${ }^{19}$ Type 2 diabetes was defined based on the WHO 1999 diagnostic criteria as FPG $\geq 7.0 \mathrm{mmol} / \mathrm{L}$ or 2 -hour plasma glucose $\geq 11.1 \mathrm{mmol} / \mathrm{L}$, while impaired fasting glucose was defined as $6.1 \mathrm{mmol} / \mathrm{L} \leq \mathrm{FPG}<7.0 \mathrm{mmol} / \mathrm{L}$, and normal condition was defined as FPG $<6.1 \mathrm{mmol} / \mathrm{L} .^{20}$ eGFR was calculated using the Modification of Diet in Renal Disease formula ${ }^{21}: 186 \times($ serum creatinine [mg/ $\mathrm{dL}])-1.154 \times$ (age) $-0.203 \times(0.742$ [if female] $)$. According to the Kidney Disease Outcomes Quality Initiative clinical practice guideline, an eGFR $<60 \mathrm{~mL} / \mathrm{min} / 1.73 \mathrm{~m}^{2}$, proteinuria and haematuria were defined as markers of chronic kidney disease $(\mathrm{CKD}) .{ }^{22}$ Urine proteinuria was recorded as negative (-), trace, $1+, 2+$ and $3+$. Albuminuria was defined as $\geq 1+$.

\section{Data collection}

Subjects participating in the study attended the medical centre in the morning after overnight fasting for at least 12 hours. After a 5 min rest, sitting BP was measured on the right arm by a trained medical staff using an electronic BP monitor. The resting BP was measured three times with 5 min intervals between them, and then the average was calculated, which was used for further analysis. Blood samples were obtained on their arrival at the medical centre and fasting glucose (FPG) was measured using the hexokinase method. TC, LDL-C, HDL-C, TG, blood urea nitrogen (BUN), creatinine and SUA were measured in an automated bioanalyser (Hitachi, Tokyo, Japan). Midstream urine specimen was collected for urinary analysis using the dipstick method. Urine $\mathrm{pH}$ and proteinuria were recorded as categorical data. Laboratory reagents were purchased from Nanjing Jiancheng Bioengineering Institute (Nanjing, China).

After blood and urine sampling, basic characteristics and medical history were collected by medical staff. Anthropometric measurements including height and body weight were obtained according to a standardised protocol. Renal ultrasonography scanning was performed and measured by an experienced radiologist who was blinded to the subjects' medical information using the GE LOGIQ P5 scanner (GE Healthcare, Milwaukee, USA). 


\section{Patient and public involvement}

No patient was involved in the design or conduct of the study, but the results of the study will be shared to patients coming for follow-up.

\section{Statistical analysis}

We divided the subjects into gender-specific quartiles according to SUA level (male-Q1: $\leq 4.9$, Q2: 5.0-5.9, Q3: 6.0-6.9, Q4: $\geq 7.0 \mathrm{mg} / \mathrm{dL}$; female-Q1: $\leq 3.9$, Q2: 4.0-4.9, Q3: $5.0-5.9, Q 4: \geq 6.0 \mathrm{mg} / \mathrm{dL})$. Distribution of variables was evaluated by the Kolmogorov-Smirnov test, and homogeneity of variance was assessed by the Levene test. The normally distributed data are reported in mean \pm SD. Skewed or non-normally distributed data are presented in median with IQR. Categorical variables are shown in percentages. Univariate analysis of variance was used to analyse the differences among the groups' means in case of normal data distribution or after logarithmic normalisation in case of skewed data (if appropriate). Kruskal-Wallis test was used to analyse the differences among the groups' medians in case of non-parametric data distribution. Differences in proportion between the groups were tested using $\mathrm{X}^{2}$ tests. If the results show differences between the groups, post-hoc tests would be done. As for the post-hoc test of normally distributed data, we used the least significance difference test if the variance was homogeneous and the Tamhane's T2 test if not. The post-hoc test of non-normally distributed data was done using the Kruskal-Wallis test. As for the post-hoc test of categorical variables, we used $\mathrm{X}^{2}$ tests. Correlations were Pearson's or Spearman's depending on the distribution of the data. In the present study, SUA, age, BMI, SBP, DBP, FPG, TC, TG, HDL-C, LDL-C and eGFR were normally distributed. Thus, Pearson's correlation was used among these variables. If Pearson's correlation analysis was statistically significant, multiple linear regression analysis was performed to determine the association of SUA with various independent variables. We analysed multiple collinearity by calculating the correlation coefficient matrix, tolerance and variance inflation factor of independent variables. Multivariable logistic regression analysis (unadjusted and full-adjusted) was used to calculate the OR for hyperuricaemia according to the different status of clinical parameters. Furthermore, multivariable logistic regression analysis (multiple adjusted models) was used to examine the association between related diseases and the SUA categories of Q2 or greater compared with the lowest SUA category. The association between hyperuricaemia and clustered CRFs had been calculated. Statistical analyses were performed by IBM SPSS Statistics V.20.0. Statistical significance was set at $\mathrm{p}$ values $<0.05$.

\section{RESULTS}

Clinical characteristics of participants in quartiles of SUA level

A total of 26768 participants with a mean age of $48.93 \pm 15.47$ years, $15041(56.2 \%)$ men and 11727
(43.8\%) women, completed the study. The prevalence rates of hyperuricaemia in men and women were $22.2 \%$ (95\% CI $21.5 \%$ to $22.9 \%$ ) and $10.8 \%$ (95\% CI $10.3 \%$ to $11.4 \%$ ), respectively. Female individuals with higher level of SUA were older than male individuals. With increasing quartiles of SUA, participants had more CRFs (obesity, hypertension and dyslipidaemia) and renal diseases (CKD and nephrolithiasis), as well as elevated levels of BMI, SBP, DBP, TC, TG, LDL-C, creatinine and BUN, and decreased levels of HDL-C and eGFR, in both men and women (table 1 ; all $\mathrm{p}$ values for trend $<0.001$ ).

\section{Prevalence of CRFs and renal diseases in quartiles of SUA level}

As demonstrated in figure 1 , there was a significant increase in the prevalence of CRFs and renal disorders across SUA quartiles in men and women (all $p$ values for trend $<0.001$ ). In male individuals with hyperuricaemia, the prevalence rates of obesity, hypertension, diabetes mellitus, dyslipidaemia, CKD and nephrolithiasis were $24.1 \%$ (95\% CI $22.6 \%$ to $25.5 \%$ ), $36.5 \%$ (95\% CI $34.9 \%$ to $38.2 \%$ ), $4.9 \%$ (95\% CI $4.2 \%$ to $5.7 \%$ ), $54.4 \%$ (95\% CI $52.7 \%$ to $56.1 \%$ ), $6.9 \%$ (95\% CI $6.1 \%$ to $7.8 \%$ ) and $18.6 \%$ (95\% CI $17.3 \%$ to $19.9 \%$ ), respectively. In female individuals with hyperuricaemia, the prevalence rates of obesity, hypertension, diabetes mellitus, dyslipidaemia, CKD and nephrolithiasis were $24.0 \%$ (95\% CI 21.6\% to $26.3 \%$ ), $43.2 \%$ (95\% CI $40.5 \%$ to $46.0 \%$ ), $10.2 \%$ (95\% CI $8.6 \%$ to $11.9 \%$ ), $45.5 \%$ (95\% CI $42.8 \%$ to $48.3 \%$ ), $12.8 \%$ (95\% CI $11.0 \%$ to $14.7 \%$ ) and $16.9 \%$ (95\% CI $14.8 \%$ to $19.0 \%)$, respectively.

\section{Correlation between SUA and various clinical parameters}

In the present study, SUA, age, BMI, SBP, DBP, FPG, TC, TG, HDL-C, LDL-C and eGFR were normally distributed. Thus, we used Pearson's correlation analysis to investigate the relationships, and the results are shown in table 2. In men, the level of SUA was positively correlated with BMI, SBP, DBP, TC, TG and LDL-C, and negatively correlated with age, FPG, HDL-C and eGFR (all $p$ values $<0.001$ ). In women, the level of SUA was positively correlated with age, BMI, SBP, DBP, FPG, TC, TG and LDL-C, and negatively correlated with HDL-C and eGFR (all p values $<0.001$ ).

Multiple linear regression analysis in table 3 showed that, adjusting for various factors, SUA was still positively correlated with BMI, SBP, TC and TG and negatively correlated with age, FPG, HDL-C and eGFR in men (all $\mathrm{p}$ values $<0.001)$. SUA was positively correlated with BMI, SBP, TC and TG and negatively correlated with FPG, HDL-C and eGFR in women (all $p$ values $<0.001$, except FPG, $\mathrm{p}=0.003$ ).

Association between hyperuricaemia and the clinical outcome Multivariable logistic regression models (unadjusted and full-adjusted) were analysed, and the results are shown in table 4, with the OR for hyperuricaemia according to different clinical outcomes. We found that after adjustment for confounders, increased levels of BMI, BP, TG, 


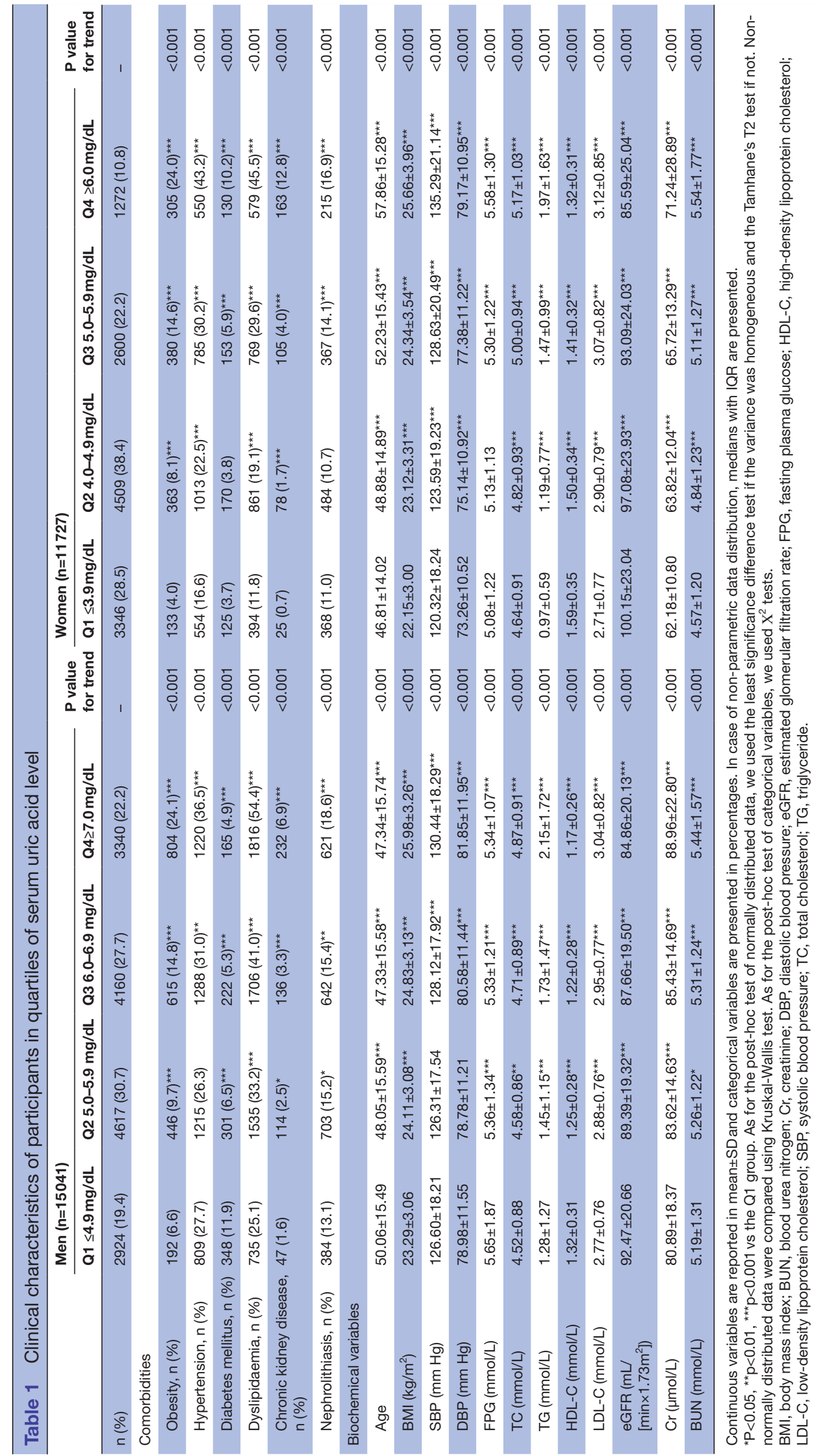




\section{$\checkmark$ Males \\ Females}

A

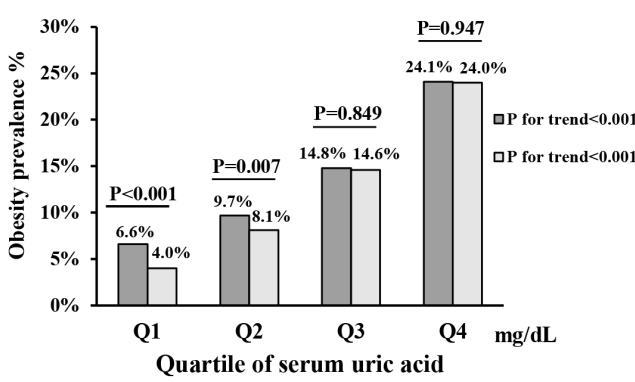

C

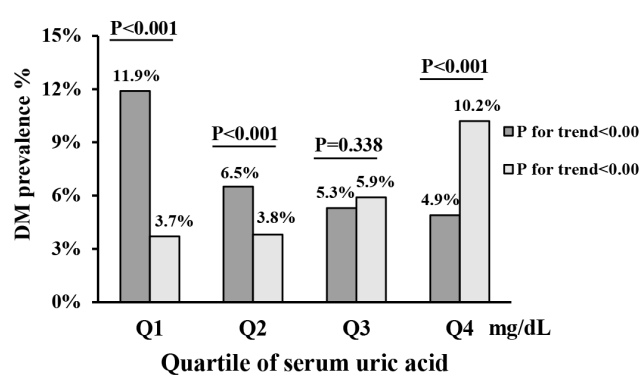

E

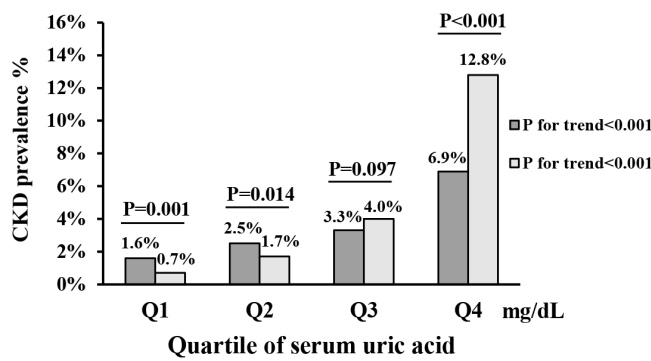

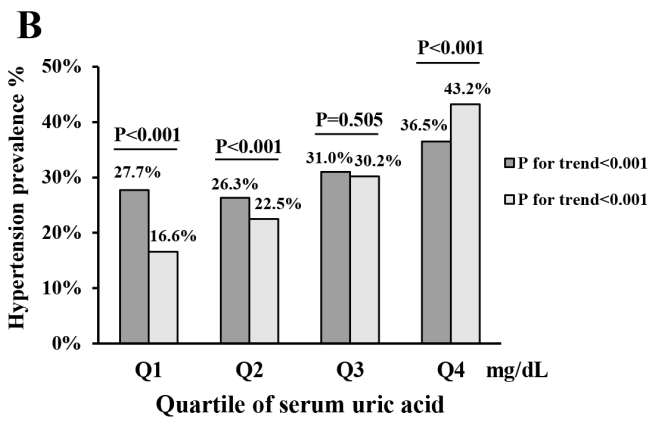

D

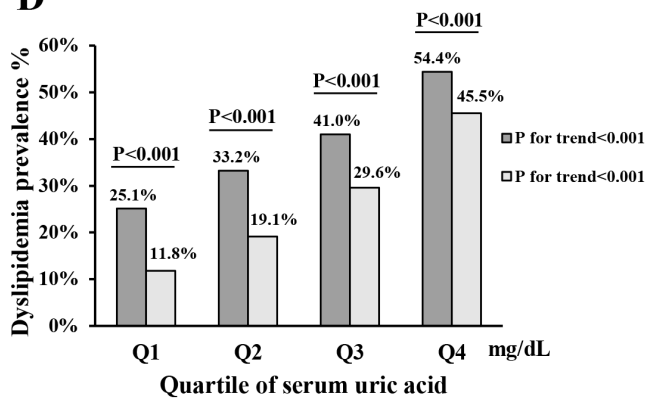

F

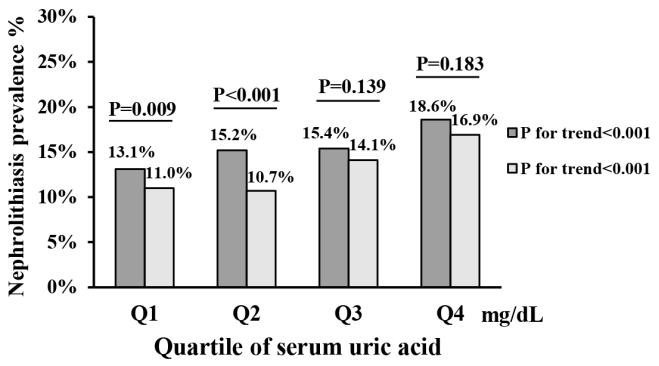

Figure 1 Prevalence of major cardiovascular disease risk factors and renal diseases in different levels of serum uric acid. Prevalence of (A) obesity, (B) hypertension, (C) diabetes mellitus (DM), (D) dyslipidaemia, (E) chronic kidney disease (CKD) and $(F)$ nephrolithiasis in different levels of serum uric acid. Gender-specific quartiles of uric acid $(\mathrm{mg} / \mathrm{dL})$ : Q1: male $\leq 4.9$, female $\leq 3.9$; Q2: male 5.0-5.9, female 4.0-4.9; Q3: male 6.0-6.9, female 5.0-5.9; Q4: male $\geq 7.0$, female $\geq 6.0$.

LDL-C and albuminuria and decreased levels of HDL-C all are positively related to increased OR of hyperuricaemia. Renal insufficiency, acid urine and nephrolithiasis also positively correlated with hyperuricaemia; however, FPG and alkaline urine negatively correlated with hyperuricaemia in both genders (all $\mathrm{p}$ values $<0.05$ ). These results suggested that individuals with overweight/ obesity, hypertension, dyslipidaemia, renal insufficiency, massive albuminuria, acid urine and nephrolithiasis were more susceptible to hyperuricaemia in both men and women.

\section{Relationship between different levels of SUA and CRFs and renal disorders}

Multivariable logistic regression analysis (multiple adjusted models) was studied, and the results are shown in table 5. The OR for obesity, hypertension, diabetes mellitus, dyslipidaemia, CKD and nephrolithiasis in the highest SUA quartile was 3.165 (95\% CI 2.644 to $3.790, \mathrm{p}<0.001$, model 3), 1.341 (95\% CI 1.181 to 1.524 , $\mathrm{p}<0.001$, model 3), 0.206 (95\% CI 0.165 to $0.257, \mathrm{p}<0.001$, model 3), 2.490 (95\% CI 2.213 to 2.801, $\mathrm{p}<0.001$, model 3), 7.081 (95\% CI 4.985 to $10.058, \mathrm{p}<0.001$, model 3 ) and 1.469 (95\% CI 1.261 to $1.710, \mathrm{p}<0.001$, model 3 ) compared with that in the lowest SUA quartile, respectively, in men.

The data in table 6 show the multivariable logistic regression analysis in women, and the OR for obesity, hypertension, diabetes mellitus, dyslipidaemia, CKD and nephrolithiasis in the highest SUA quartile was 3.776 (95\% CI 2.969 to $4.802, \mathrm{p}<0.001$, model 3), 1.289 (95\% CI 1.076 to $1.545, \mathrm{p}=0.006$, model 3 ), 0.524 (95\% CI 0.384 to $0.715, \mathrm{p}<0.001$, model 3 ), 3.614 (95\% CI 3.058 to 4.272 , $\mathrm{p}<0.001$, model 3), 11.571 (95\% CI 7.267 to 18.423 , $\mathrm{p}<0.001$, model 3) and 1.242 (95\% CI 1.009 to 1.530 , 
Table 2 Correlation coefficients between serum uric acid and various parameters

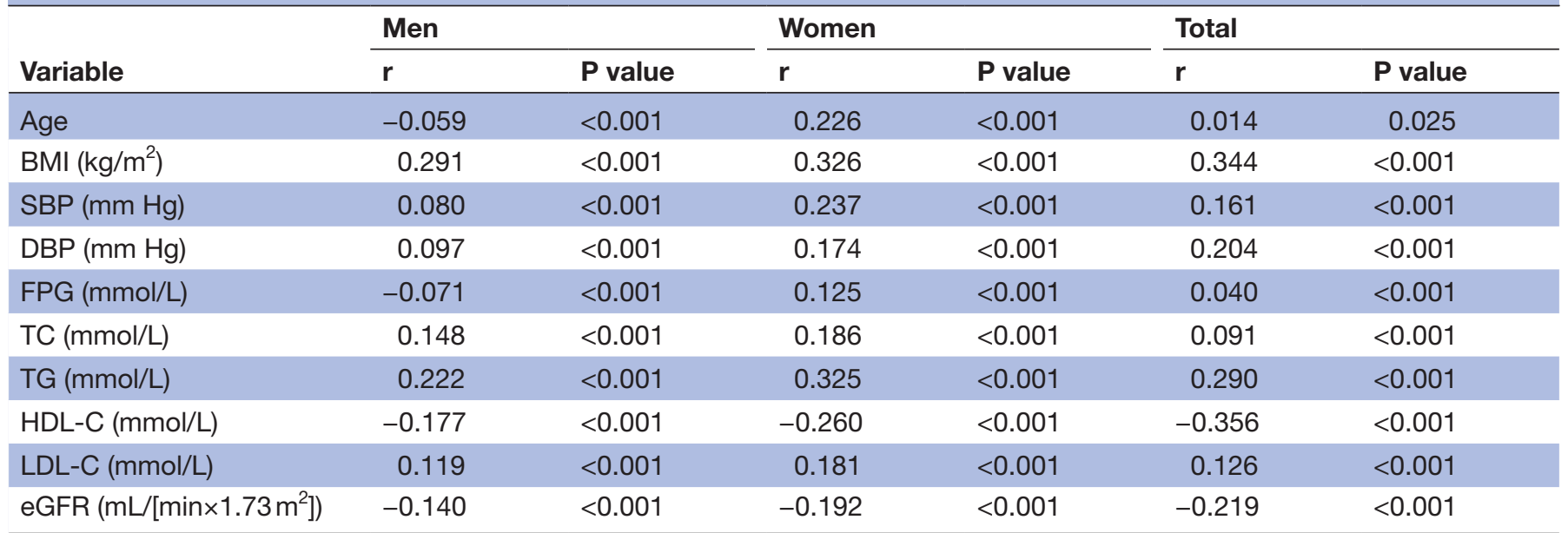

BMI, body mass index; DBP, diastolic blood pressure; eGFR, estimated glomerular filtration rate; FPG, fasting plasma glucose; HDL-C, highdensity lipoprotein cholesterol; LDL-C, low-density lipoprotein cholesterol; SBP, systolic blood pressure; TC, total cholesterol; TG, triglyceride.

$\mathrm{p}=0.041$, model 3) compared with those in the lowest SUA quartile, respectively.

From multivariable logistic regression analysis in men and women, we concluded that hyperuricaemia positively correlated with obesity, hypertension, dyslipidaemia, CKD and nephrolithiasis but negatively correlated with diabetes mellitus (all $\mathrm{p}<0.05)$ in both genders. Furthermore, women had stronger association between hyperuricaemia and CKD than men.

\section{Association between hyperuricaemia and clustered CRFs}

Multivariable logistic regression was analysed for hyperuricaemia and clustered CRFs, and the results are shown in table 7. After adjustment for age, compared with the group of zero CRF reference group, the OR for group of $\geq 3$ CRFs was $3.889(95 \%$ CI 3.322 to $4.552, \mathrm{p}<0.001)$ and $6.270(95 \%$ CI 4.936 to $7.964, \mathrm{p}<0.001)$ in men and women, respectively. The individuals with more CRFs were associated with higher ORs of hyperuricaemia both in men and women ( $p$ for trend $<0.001$ ). Furthermore, women had a stronger association of hyperuricaemia with clustered CRFs than men.

\section{DISCUSSION}

In the present study, we found that increasing prevalence of CRFs and renal disorders was predominantly associated with increased SUA levels in Shanghai population. According to our multicentre epidemiological study of 26768 subjects, the prevalence of hyperuricaemia in Shanghai was $17.2 \%$ (95\% CI $16.8 \%$ to $17.7 \%), 22.2 \%$

Table 3 Multiple linear regression analysis of the association of various independent variables with serum uric acid (dependent variable)

\begin{tabular}{|c|c|c|c|c|c|c|}
\hline \multirow[b]{2}{*}{ Variable } & \multicolumn{2}{|l|}{ Men } & \multicolumn{2}{|l|}{ Women } & \multicolumn{2}{|l|}{ Total } \\
\hline & St B & $P$ value & St B & $P$ value & St B & $P$ value \\
\hline Age & -0.127 & $<0.001$ & & & -0.142 & $<0.001$ \\
\hline SBP $(m m ~ H g)$ & 0.066 & $<0.001$ & 0.059 & $<0.001$ & 0.092 & $<0.001$ \\
\hline FPG (mmol/L) & -0.122 & $<0.001$ & -0.027 & 0.003 & -0.059 & $<0.001$ \\
\hline TG (mmol/L) & 0.114 & $<0.001$ & 0.147 & $<0.001$ & 0.108 & $<0.001$ \\
\hline HDL-C (mmol/L) & -0.084 & $<0.001$ & -0.168 & $<0.001$ & -0.252 & $<0.001$ \\
\hline eGFR (mL/[min $\left.\left.\times 1.73 \mathrm{~m}^{2}\right]\right)$ & -0.154 & $<0.001$ & -0.145 & $<0.001$ & -0.208 & $<0.001$ \\
\hline
\end{tabular}

Multicollinearity analysis showed that SBP and DBP highly correlate with each other, so do TC and LDL-C. Backward elimination was adopted for multiple linear regression to identify independent variables which have the most impact on dependent variables. Finally, the independent variables DBP and LDL-C were removed from male and from the total. The independent variables DBP, LDL-C and age were removed from female.

Standardised beta coefficients $(S t B)$ refer to how many SD a dependent variable will change per SD increase in the predictor variable. BMI, body mass index; DBP, diastolic blood pressure; eGFR: estimated glomerular filtration rate; FPG, fasting plasma glucose; HDL-C, highdensity lipoprotein cholesterol; LDL-C, low-density lipoprotein cholesterol; SBP, systolic blood pressure; TC, total cholesterol; TG, triglyceride. 


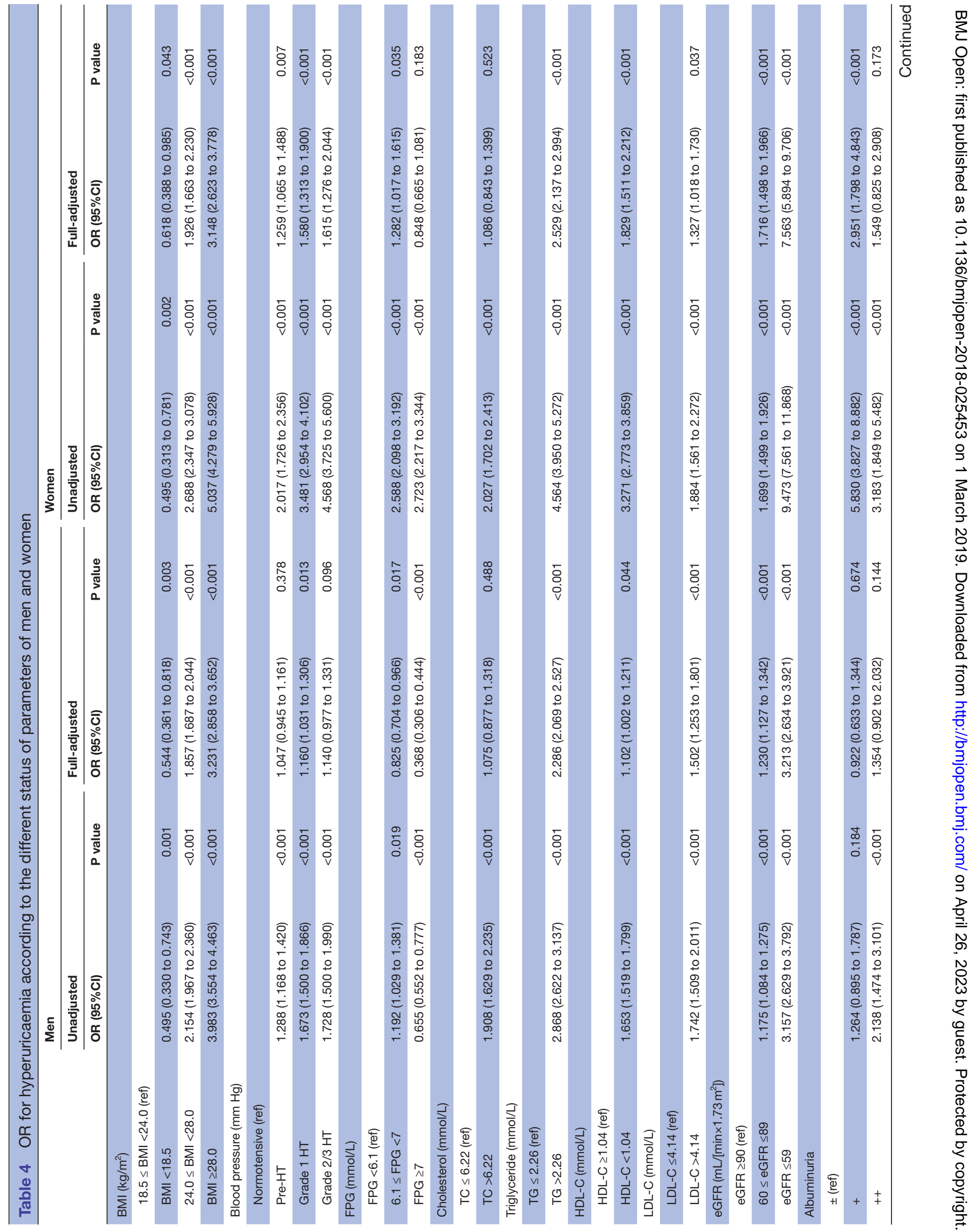




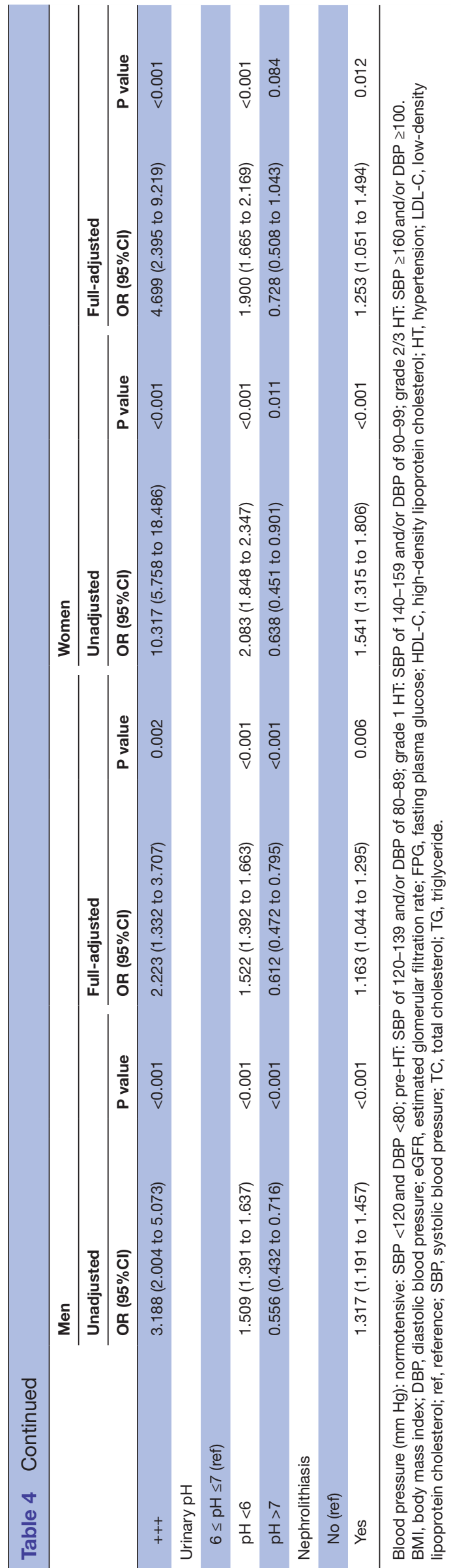

(95\% CI $21.5 \%$ to $22.9 \%$ ) in men and $10.8 \%$ (95\% CI $10.3 \%$ to $11.4 \%)$ in women. This is higher than the prevalence of hyperuricaemia among nationwide Chinese adults between 2009 and $2010(8.4 \%),{ }^{23}$ and close to the developed countries such as the USA $(21.4 \%)$ and Japan $(25.8 \%) .{ }^{24}{ }^{25}$ Along with the rapid economic development of Shanghai, the consumption of purine-rich food and alcohol is increasing. Meanwhile, obesity and ageing population are rising. Changes in lifestyle, dietary habit and population are attributed to this phenomenon. The gender-related differences between SUA level and age could be due to sex hormones. Oestrogen promoted urinary UA excretion. ${ }^{26}$

Obesity is probably the major risk factor for CVD. Obesity is accompanied by increased waist circumference, hyperinsulinaemia and dyslipidaemia. The most frequent manifestation of glucose metabolism in obese population is hyperinsulinaemia, a compensatory mechanism, whereas fasting glucose remains normal. ${ }^{27}$ It is likely that the presence of insulin resistance and hyperinsulinaemia facilitates renal tubular cells to reabsorb sodium coupling with urate. ${ }^{28}$ This may be the reason why obesity is accompanied by hyperuricaemia. Our data are also in agreement with the results from two retrospective studies among youths. ${ }^{29} 30$

Hypertension is another important risk factor for CVD. Recently, Borghi et $a l^{31-33}$ found that SUA levels were significantly higher in patients with untreated and poorly controlled hypertension in comparison with normotensive controls and patients with well-controlled hypertension. Our data were in accordance with Borghi et $a l^{81-33}$ results. After adjustment of confounding factors, hypertension was still significantly associated with hyperuricaemia. The OR for hypertension in the highest SUA quartile was $1.341(95 \%$ CI 1.181 to $1.524, \mathrm{p}<0.001)$ in men and 1.289 (95\% CI 1.076 to $1.545, \mathrm{p}=0.006)$ in women. Future follow-up studies and randomised clinical trials are required to investigate the causative relationships between urate and BP. However, animal studies had shown that a high level of UA caused high BP, which was mediated by the activation of renal and systemic reninangiotensin-aldosterone systems (RAAS), oxidative stress, vascular insulin resistance and loss of endothelial nitric oxide. ${ }^{3435}$

One of the interesting findings in our investigation was that the higher level of SUA was positively correlated with the higher prevalence rates of diabetes mellitus in women, but inversely correlated with that in men (figure 1). Pearson's correlation analysis demonstrated that the level of SUA positively correlated with FPG in women, but negatively in men. However, the coefficients of determination of the models were low. The obtained $\mathrm{p}$ values below 0.001 may be attributed to the effect of the big sample size. So we further adopted multiple linear regression and multivariable logistic regression analysis for association of various variables with SUA. After adjustment of confounding factors, the results of multivariable logistic regression showed inverse association of 


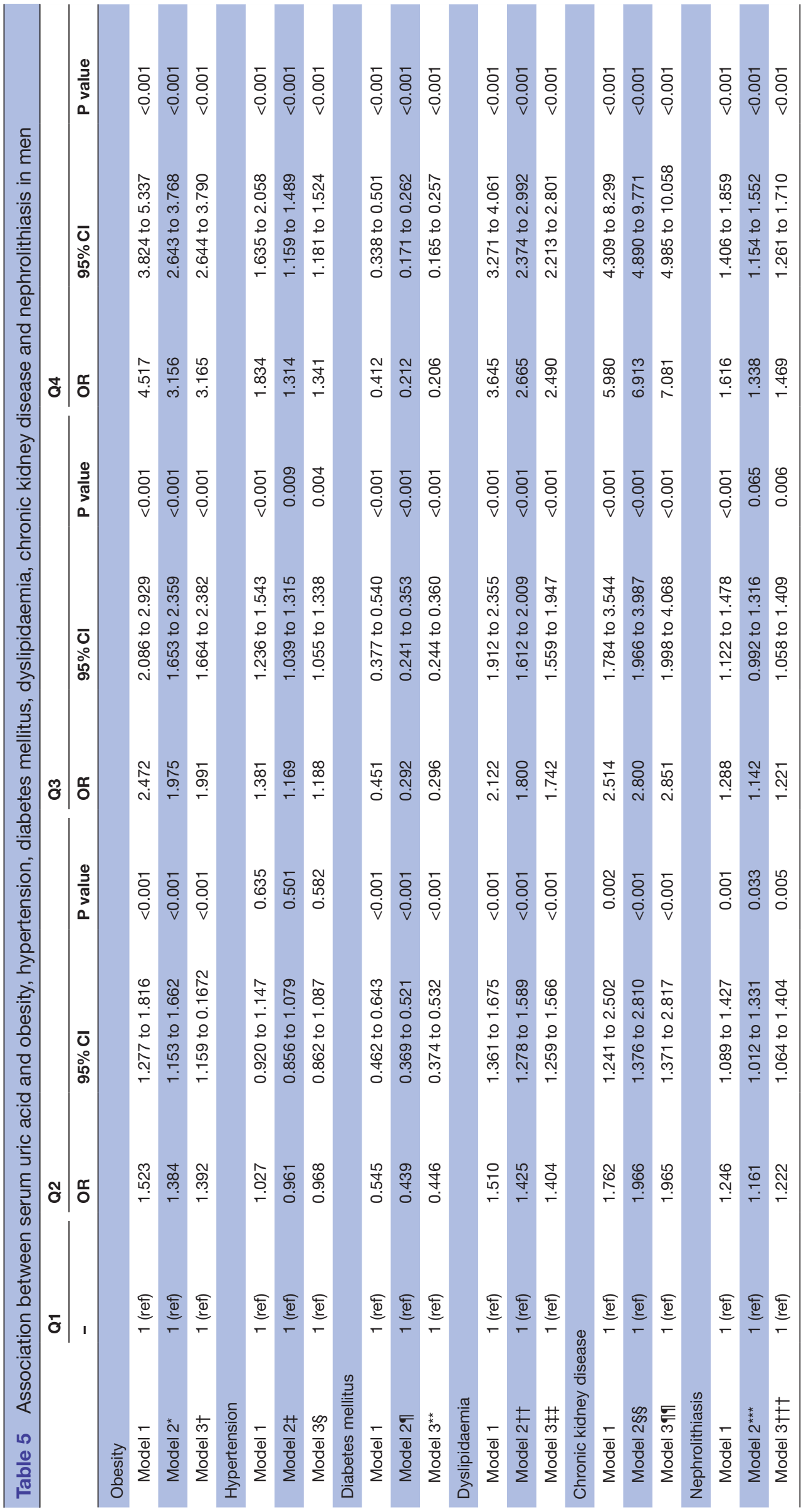

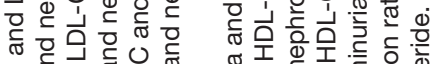
O

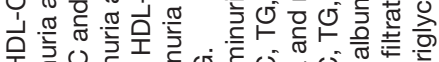

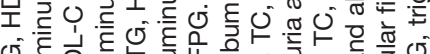

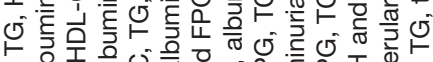

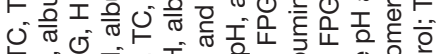

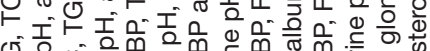
ण์

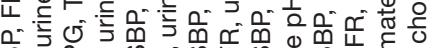

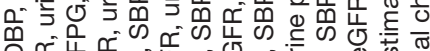

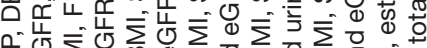

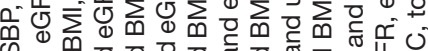

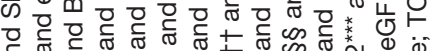

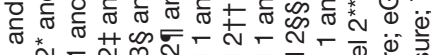

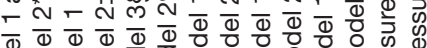

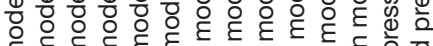

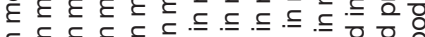

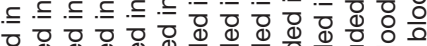
บny (1) (1)III

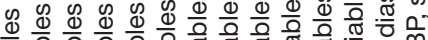

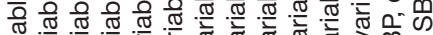

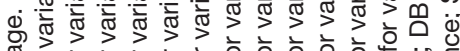

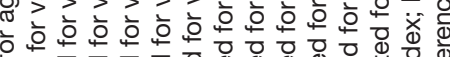

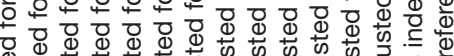

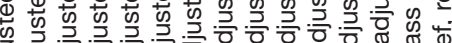




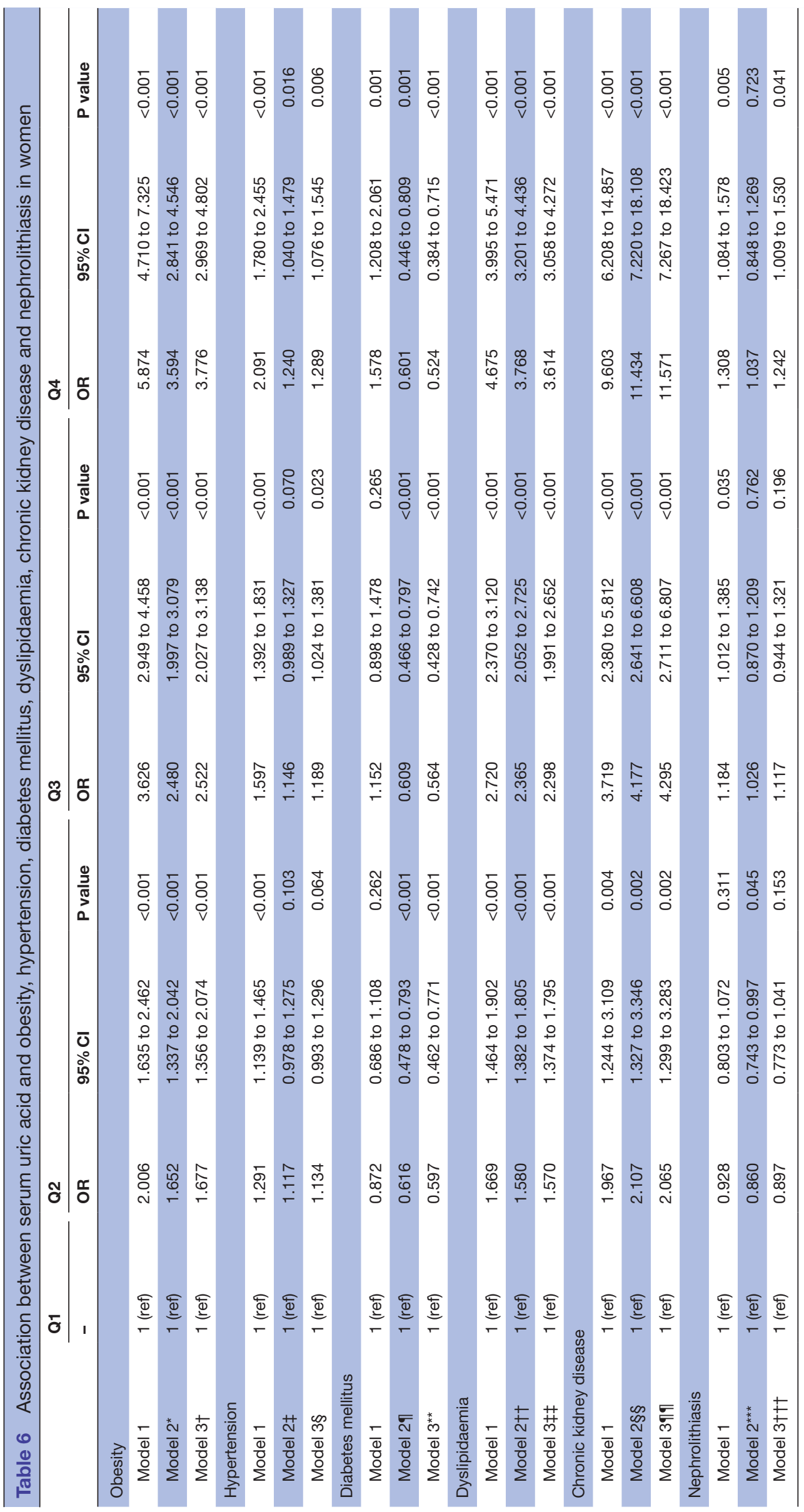

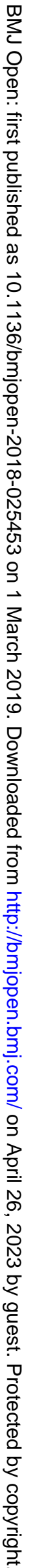


SUA with diabetes mellitus in both men and women. The OR for diabetes mellitus in the highest SUA quartile was 0.206 (95\% CI 0.165 to $0.257, \mathrm{p}<0.001$ ) in men and 0.524 $(95 \%$ CI 0.384 to $0.715, \mathrm{p}<0.001)$ in women. This was also confirmed by multiple linear regression analysis. We speculated that this phenomenon is probably due to the presence of high level of blood glucose, which promotes renal excretion of SUA. It has been indicated that hyperglycaemia worsens the function of beta cells and deteriorates glycaemic control, which gradually elevate the rate of renal glomerular filtration. ${ }^{36}$ Hyperfiltration due to multiple kidney disorders will lead to increased excretion of UA and will be more susceptible to diabetic nephropathy with decreasing eGFR. ${ }^{37}$

Based on our data, hyperuricaemia had a remarkable association with CRFs and renal disorders. The more CRFs individuals had, the higher ORs of hyperuricaemia were observed in both genders, especially in women. This was consistent with Borghi et $a l \mathrm{~s}^{3}{ }^{38}$ studies that SUA was associated with cardiovascular risk score, and the results supported an independent association between SUA and CVD. There were numerous mechanisms involved in the close association between SUA and CVDs. ${ }^{39-42}$ UA stimulates platelet-derived growth factor receptor- $\beta$ phosphorylation, leading to vascular smooth muscle cell proliferation. ${ }^{39} \mathrm{UA}$ increases oxidative stress and stimulates the activation of renin-angiotensin system, resulting in the senescence and apoptosis of human umbilical vein endothelial cells. ${ }^{40} \mathrm{UA}$ also can cause mitochondrial alterations and decrease intracellular ATP production and subsequently result in endothelial dysfunction in human aortic endothelial cells. ${ }^{41}$ A large quantity of animal experiments and human epidemiological documents indicated that SUA-lowering treatment was beneficial for CVDs. ${ }^{43-47}$

On the other hand, the related mechanisms in the link between hyperuricaemia and CKD were not well investigated. It was demonstrated that $70 \%$ of urate eliminated occurs in the kidneys, and decreased eGFR would result in elevated levels of SUA. ${ }^{48}$ However, UA could induce oxidative stress, trigger activation of RAAS and inflammation, cause endothelial dysfunction, and thus subsequently lead to the decline in eGFR. ${ }^{354950}$ The persistently high level of SUA predicts the high risk of developing CKD. ${ }^{51}$ There was a marked association of SUA with albuminuria in patients with renal insufficiency. ${ }^{52}$ Our study was consistent with many prospective studies, ${ }^{1453} 54$ showing that SUA is a significant risk factor for CKD and proteinuria, which is independent of confounders of CRFs. We further demonstrated that hyperuricaemia significantly correlated with acid urine and nephrolithiasis, which was confirmed by the prevalence rates of CKD and nephrolithiasis across the SUA levels.

This study has some limitations that need to be mentioned. First, it was a cross-sectional study and the results could not establish causative relationships between hyperuricaemia and CRF clustering and renal diseases. Future follow-up studies are required for more accurate evaluation of these relationships. Second, the 
data were from three medical centres' databases which lacked details on waist circumference, homeostasis model assessment of insulin resistance, smoking, drinking, lifestyles, diet and pharmacotherapy, which might affect deviations in some clinical outcomes. Nonetheless, the strengths of our study included its strict exclusion criteria based on medical histories and laboratory findings, and we conducted a multicentre study with a large sample size which ensured sufficient parameters and accurate results. The relationship was analysed in both genders, and a solid conclusion on the differences between men and women was gained.

\section{CONCLUSION}

In summary, our data demonstrated that the increasing rate of prevalence of CRFs and renal disorders was significantly correlated with the elevated level of SUA. Hyperuricaemia was remarkably linked with CVD-related risk factors and kidney disease, especially in women.

\section{Author affiliations}

${ }^{1}$ Department of Nephrology, Shanghai East Hospital, Tongji University School of Medicine, Shanghai, China

${ }^{2}$ Department of Internal Medicine, Pudong New District Gongli Hospital, Shanghai, China

${ }^{3}$ Department of Nephrology, Baoshan Branch of First People's Hospital, Shanghai Jiaotong University, Shanghai, China

${ }^{4}$ Key Laboratory of Arrhythmias of the Ministry of Education of China, Research Center for Translational Medicine, Shanghai East Hospital, Tongji University School of Medicine, Shanghai, China

${ }^{5}$ Department of Medical Laboratory, Pudong New District Gongli Hospital, Shanghai, China

${ }^{6}$ Department of Medicine, Department of Medicine, Rhode Island Hospital and Brown University School of Medicine, Providence, Rhode Island, USA

${ }^{7}$ Department of Nephrology, Shanghai East Hospital, Tongji University School of Medicine, Shanghai, China

Acknowledgements The authors appreciate all the participants and their families. They also thank the members of the study team from Shanghai East Hospital Affiliated to Tongji University School of Medicine, Pudong New District Gongli Hospital and Baoshan Branch of Shanghai First People's Hospital for their assistance in completing this project.

Contributors MT, NL, SZ, YS and YZ performed the statistical analysis and wrote the manuscript. MT, XM, XP, YC, HG and YS participated in the data collection. MT, $\mathrm{NL}, \mathrm{SZ}$ and YZ contributed to the discussion. MT and NL participated in the design of the study and edited the manuscript. All authors have read and approved the final manuscript. The corresponding author had full access to all the data in the study and had final responsibility for the decision to submit for publication.

Funding This study was also supported by the National Nature Science Foundation of China grants (81670690, 81470991 and 81200492 to NL, 81830021, 81670623, 81470920 and 81270778 to SZ), the Key Discipline Construction Project of Pudong Health Bureau of Shanghai (PWZxk2017-05 to NL), the Branch Grant of National Key Grants of the Ministry of Science and Technology (2018YFA0108802 to SZ), US National Institutes of Health (2R01DK08506505A1 to SZ), the Shanghai Scientific Committee of China (13PJ1406900 to NL), and Shanghai Health Bureau grant (20134464 to XP).

\section{Competing interests None declared.}

Patient consent for publication Obtained.

Ethics approval This study was approved by the Human Research Ethics Committee of the Shanghai East Hospital Affiliated to Tongji University School of Medicine, the Human Research Ethics Committee of Pudong New District Gongli Hospital and the Human Research Ethics Committee of Baoshan Branch of Shanghai First People's Hospital. All methods were performed in compliance with
Good Clinical Practice (GCP) guidelines and the Declaration of Helsinki. Written informed consent was obtained from each participant before data collection.

Provenance and peer review Not commissioned; externally peer reviewed.

Data sharing statement The data sets generated and analysed during the current study are available from the corresponding author upon reasonable request.

Open access This is an open access article distributed in accordance with the Creative Commons Attribution Non Commercial (CC BY-NC 4.0) license, which permits others to distribute, remix, adapt, build upon this work non-commercially, and license their derivative works on different terms, provided the original work is properly cited, appropriate credit is given, any changes made indicated, and the use is non-commercial. See: http://creativecommons.org/licenses/by-nc/4.0/.

\section{REFERENCES}

1. Jalal DI. Hyperuricemia, the kidneys, and the spectrum of associated diseases: a narrative review. Curr Med Res Opin 2016;32:1863-9.

2. Kanbay M, Jensen T, Solak $\mathrm{Y}$, et al. Uric acid in metabolic syndrome: From an innocent bystander to a central player. Eur J Intern Med 2016;29:3-8.

3. Borghi C, Rosei EA, Bardin T, et al. Serum uric acid and the risk of cardiovascular and renal disease. J Hypertens 2015;33:1729-41.

4. Kuwabara M, Bjornstad P, Hisatome I, et al. Elevated serum uric acid level predicts rapid decline in kidney function. Am J Nephrol 2017;45:330-7.

5. Gertler MM, Garn SM, Levine SA. Serum uric acid in relation to age and physique in health and in coronary heart disease. Ann Intern Med 1951;34:1421-31.

6. Wang TT, Lin B, Cui WX, et al. Clustering of Cardiovascular Risk Factors and Diabetes: A Prospective Cohort Study on the Inner Mongolian Population in China. Biomed Environ Sci 2018;31:749-56.

7. de Souza Filho ZA, Ferreira AA, Dos Santos J, et al. Cardiovascular risk factors with an emphasis on hypertension in the Mura Indians from Amazonia. BMC Public Health 2018;18:1251.

8. Matthews KA, Jennings JR, Lee L, et al. Depressive symptoms during childhood and cardiovascular risk factors in black and white men. Psychosom Med 2018.

9. van Sloten TT, Tafflet M, Périer MC, et al. Association of Change in Cardiovascular Risk Factors With Incident Cardiovascular Events. JAMA 2018;320:1793-804.

10. Lipkowitz MS. Regulation of uric acid excretion by the kidney. Curr Rheumatol Rep 2012;14:179-88.

11. DiBianco JM, Jarrett TW, Mufarrij P. Metabolic syndrome and nephrolithiasis risk: Should the medical management of nephrolithiasis include the treatment of metabolic syndrome?. Rev Urol 2015;17:117-28.

12. Jalal DI, Rivard CJ, Johnson RJ, et al. Serum uric acid levels predict the development of albuminuria over 6 years in patients with type 1 diabetes: findings from the Coronary Artery Calcification in Type 1 Diabetes study. Nephrol Dial Transplant 2010;25:1865-9.

13. Klisic A, Kocic G, Kavaric N, et al. Xanthine oxidase and uric acid as independent predictors of albuminuria in patients with diabetes mellitus type 2. Clin Exp Med 2018;18:283-90.

14. Bellomo G, Venanzi S, Verdura C, et al. Association of uric acid with change in kidney function in healthy normotensive individuals. $A m \mathrm{~J}$ Kidney Dis 2010;56:264-72.

15. Mwasongwe SE, Fülöp T, Katz R, et al. Relation of uric acid level to rapid kidney function decline and development of kidney disease: The Jackson Heart Study. J Clin Hypertens 2018;20:775-83.

16. Becker MA, Schumacher HR, Wortmann RL, et al. Febuxostat compared with allopurinol in patients with hyperuricemia and gout. $N$ Engl J Med 2005;353:2450-61.

17. WHO Expert Consultation. Appropriate body-mass index for Asian populations and its implications for policy and intervention strategies. Lancet 2004;363:157-63.

18. National High Blood Pressure Education P. The Seventh Report of the Joint National Committee on Prevention, Detection, Evaluation, and Treatment of High Blood Pressure. Bethesda (MD): National Heart, Lung, and Blood Institute (US), 2004.

19. Joint Committee for Developing Chinese guidelines on Prevention and Treatment of Dyslipidemia in Adults. [Chinese guidelines on prevention and treatment of dyslipidemia in adults]. Zhonghua Xin Xue Guan Bing Za Zhi 2007;35:390-419.

20. Alberti KG, Zimmet PZ. Definition, diagnosis and classification of diabetes mellitus and its complications. Part 1: diagnosis and classification of diabetes mellitus provisional report of a WHO consultation. Diabet Med 1998;15:539-53. 
21. Levey AS, Bosch JP, Lewis JB, et al. A more accurate method to estimate glomerular filtration rate from serum creatinine: a new prediction equation. Modification of Diet in Renal Disease Study Group. Ann Intern Med 1999;130:461-70.

22. National Kidney Foundation. K/DOQI clinical practice guidelines for chronic kidney disease: evaluation, classification, and stratification. Am J Kidney Dis 2002;39:S1-266.

23. Liu H, Zhang XM, Wang YL, et al. Prevalence of hyperuricemia among Chinese adults: a national cross-sectional survey using multistage, stratified sampling. J Nephrol 2014;27:653-8.

24. Zhu Y, Pandya BJ, Choi HK. Prevalence of gout and hyperuricemia in the US general population: the National Health and Nutrition Examination Survey 2007-2008. Arthritis Rheum 2011;63:3136-41.

25. Nagahama K, Iseki K, Inoue T, et al. Hyperuricemia and cardiovascular risk factor clustering in a screened cohort in Okinawa, Japan. Hypertens Res 2004;27:227-33.

26. Sekine T, Miyazaki H, Endou H. Molecular physiology of renal organic anion transporters. Am J Physiol Renal Physiol 2006;290:F251-61.

27. Yoo TW, Sung KC, Shin HS, et al. Relationship between serum uric acid concentration and insulin resistance and metabolic syndrome. Circ J 2005;69:928-33.

28. Bjornstad P, Lanaspa MA, Ishimoto $\mathrm{T}$, et al. Fructose and uric acid in diabetic nephropathy. Diabetologia 2015;58:1993-2002.

29. Lurbe $\mathrm{E}$, Torro Ml, Alvarez-Pitti J, et al. Uric acid is linked to cardiometabolic risk factors in overweight and obese youths. $J$ Hypertens 2018;36:1840-6.

30. Ishiro M, Takaya R, Mori Y, et al. Association of uric acid with obesity and endothelial dysfunction in children and early adolescents. Ann Nutr Metab 2013;62:169-76.

31. Cicero AF, Rosticci M, Fogacci F, et al. High serum uric acid is associated to poorly controlled blood pressure and higher arterial stiffness in hypertensive subjects. Eur J Intern Med 2017;37:38-42.

32. Mancia G, Grassi G, Borghi C, Hyperuricemia BC. Hyperuricemia, urate deposition and the association with hypertension. Curr Med Res Opin 2015;31 Suppl 2(Suppl 2):15-19.

33. Cicero AF, Salvi P, D'Addato $S$, et al. Association between serum uric acid, hypertension, vascular stiffness and subclinical atherosclerosis: data from the Brisighella Heart Study. J Hypertens 2014;32:57-64.

34. Choi YJ, Yoon Y, Lee KY, et al. Uric acid induces endothelial dysfunction by vascular insulin resistance associated with the impairment of nitric oxide synthesis. Faseb J 2014;28:3197-204.

35. Corry DB, Eslami P, Yamamoto K, et al. Uric acid stimulates vascular smooth muscle cell proliferation and oxidative stress via the vascular renin-angiotensin system. J Hypertens 2008;26:269-75.

36. Li Q, Yang Z, Lu B, et al. Serum uric acid level and its association with metabolic syndrome and carotid atherosclerosis in patients with type 2 diabetes. Cardiovasc Diabetol 2011;10:72.

37. Lytvyn Y, Škrtić M, Yang GK, et al. Glycosuria-mediated urinary uric acid excretion in patients with uncomplicated type 1 diabetes mellitus. Am J Physiol Renal Physiol 2015;308:F77-83.

38. Borghi C, Rodriguez-Artalejo F, De Backer G, et al. Serum uric acid levels are associated with cardiovascular risk score: A post hoc analysis of the EURIKA study. Int J Cardiol 2018;253:167-73.
39. Kırça M, Oğuz N, Çetin A, et al. Uric acid stimulates proliferative pathways in vascular smooth muscle cells through the activation of p38 MAPK, p44/42 MAPK and PDGFRß. J Recept Signal Transduct Res 2017:37:167-73.

40. Yu MA, Sánchez-Lozada LG, Johnson RJ, et al. Oxidative stress with an activation of the renin-angiotensin system in human vascular endothelial cells as a novel mechanism of uric acid-induced endothelial dysfunction. J Hypertens 2010;28:1234-42.

41. Sánchez-Lozada LG, Lanaspa MA, Cristóbal-García M, et al. Uric acid-induced endothelial dysfunction is associated with mitochondrial alterations and decreased intracellular ATP concentrations. Nephron Exp Nephrol 2012;121:e71-8.

42. Strazzullo P, Puig JG. Uric acid and oxidative stress: relative impact on cardiovascular risk? Nutr Metab Cardiovasc Dis 2007:17:409-14.

43. Kondo M, Imanishi M, Fukushima K, et al. Xanthine oxidase inhibition by febuxostat in macrophages suppresses angiotensin II-induced Aortic fibrosis. Am J Hypertens 2018.

44. Namai-Takahashi A, Sakuyama A, Nakamura T, et al. Xanthine oxidase inhibitor, febuxostat ameliorates the high salt intake-induced cardiac hypertrophy and fibrosis in dahl salt-sensitive rats. $\mathrm{Am} \mathrm{J}$ Hypertens 2019;32:26-33.

45. Maclsaac RL, Salatzki J, Higgins P, et al. Allopurinol and cardiovascular outcomes in adults with hypertension. Hypertension 2016:67:535-40.

46. Tani S, Nagao K, Hirayama A. Effect of febuxostat, a xanthine oxidase inhibitor, on cardiovascular risk in hyperuricemic patients with hypertension: A prospective, open-label, pilot study. Clin Drug Investig 2015;35:823-31.

47. Xin W, Mi S, Lin Z. Allopurinol therapy improves vascular endothelial function in subjects at risk for cardiovascular diseases: a meta-analysis of randomized controlled trials. Cardiovasc Ther 2016;34:441-9.

48. Marangella M. Uric acid elimination in the urine. Pathophysiological implications. Contrib Nephrol 2005;147:132-48.

49. Bao J, Shi Y, Tao M, et al. Pharmacological inhibition of autophagy by 3-MA attenuates hyperuricemic nephropathy. Clin Sci 2018;132:2299-322.

50. Maruhashi T, Hisatome I, Kihara Y, et al. Hyperuricemia and endothelial function: From molecular background to clinical perspectives. Atherosclerosis 2018;278:226-31.

51. Chou YC, Kuan JC, Yang T, et al. Elevated uric acid level as a significant predictor of chronic kidney disease: a cohort study with repeated measurements. J Nephrol 2015;28:457-62.

52. De Cosmo S, Viazzi F, Pacilli A, et al. Serum Uric Acid and Risk of CKD in Type 2 Diabetes. Clin J Am Soc Nephrol 2015;10:1921-9.

53. Takae K, Nagata M, Hata J, et al. Serum Uric Acid as a Risk Factor for Chronic Kidney Disease in a Japanese Community - The Hisayama Study. Circ J 2016;80:1857-62.

54. Li L, Yang C, Zhao Y, et al. Is hyperuricemia an independent risk factor for new-onset chronic kidney disease?: A systematic review and meta-analysis based on observational cohort studies. BMC Nephrol 2014;15:122. 